\title{
Multimode Cavity Efficiency Optimization by Optimum Load Location-Experimental Approach
}

María E. Requena-Pérez, Juan L. Pedreño-Molina, Juan Monzó-Cabrera, and Alejandro Díaz-Morcillo, Member, IEEE

\begin{abstract}
In this paper, a novel load-matching procedure for microwave-heating applicators is presented and tested. In order to accomplish the optimization procedure, an optimization method based on the use of the Lebenverg-Marquardt technique has been specifically developed and tested on two different microwave ovens. The proposed procedure allows an efficient optimization of three-dimensional microwave applicators by means of the dielectric sample relocation as a function of its complex permittivity, size, and operating frequency. Experimental measurements of the reflection coefficient are presented and analyzed over several samples and multimode cavities. Results indicate that high power efficiencies can be obtained provided that operating frequency is not just below TM-mode cutoff frequencies for the transverse dimensions of the oven.
\end{abstract}

Index Terms-Electromagnetic heating, load matching, multimode applicator, power efficiency.

\section{INTRODUCTION}

$\mathbf{I}$ NDUSTRIAL microwave-heating applications have reached maturity in several areas such as textile, paper, composite, rubber, and food industries. In these cases, the microwave applicators are very often based on multimode cavities, mainly when the materials to be processed are big when compared to the used wavelength [1]-[4]. Although the benefits for such industrial processes are enormous when applying microwaves, either solely or in combination with conventional energy sources, the design of these electromagnetic structures are still based on trial-and-error and personnel experience.

However, the decrease in the costs of software and hardware during the past years have multiplied the use of numerical methods to solve electromagnetic situations related to microwave heating technology and multimode applicators [5]. Despite the application of commercial electromagnetic software to the microwave-heating devices design [6], very few studies have tried to optimize the efficiency of microwave heating devices from an electromagnetic point-of-view [7]-[9] mainly due to the high computational cost of each iteration during the optimization loop.

Multimode microwave-heating cavities that contain high-permittivity dielectrics have usually been adapted by several means. Conventional ones require the use of irises and waveguide tuning screws acting as triple or quadruple stubs [3], [4].

Manuscript received October 1, 2004; revised December 16, 2004. This work was supported in part by the Spanish Science and Technology Ministry under Project TIC2001-2778-CO2-02.

The authors are with the Departmento de Tecnologías de la Información y las Comunicaciones, Universidad Politécnica de Cartagena, 30202 Cartagena, Murcia, Spain (e-mail: mae.requena@upct.es; juan.monzo@upct.es).

Digital Object Identifier 10.1109/TMTT.2005.848767
These elements, however, need to be finely tuned to provide an adequate match to load and frequency conditions.

In a previous study [10], a novel optimization approach was carried out through simulations within a two-dimensional microwave oven to achieve good efficiency ratios for a wide variety of loads. Three variables were considered in that study, i.e., the location, dielectric constant, and loss factor of the load. An iterative procedure was employed to find the simulated optimum location wherein the load should be placed to provide a minimum reflection coefficient.

In this paper, the previous load-matching technique has been experimentally tested for different types of loads and threedimensional microwave multimode cavities. Five variables are considered in our study, which are: 1) location; 2) permittivity; 3) shape of the load; 4) size of the load; and 5) operating frequency of the microwave source. An iterative procedure based on the Lebenverg-Marquardt (LM) method is employed to find the optimum location wherein the load must be placed to provide a minimum reflection coefficient.

\section{EXPERIMENTAL SETUP}

\section{A. Measurement Equipment}

Two different multimode ovens have been used for efficiency measurements, i.e., cavity I $\left(30 \times 30 \times 30 \mathrm{~cm}^{3}\right)$ and cavity II $\left(60 \times 60 \times 60 \mathrm{~cm}^{3}\right)$. Fig. 1 shows the diagram for cavity I. This multimode oven consists of a $30 \times 30 \times 30 \mathrm{~cm}^{3}$ aluminum cubic cavity. The sidewall of the cavity contains a centered WR-340 waveguide $\left(8.6 \times 4.3 \times 12 \mathrm{~cm}^{3}\right)$ that acts as the feeding port. In order to support and move the sample, several styrofoam supporting structures have been included. Styrofoam has been chosen for this purpose due to its low permittivity and negligible dielectric losses $\left(\varepsilon^{*}=1.03\right)$. Cavity II was constructed with the same materials and the same structure as cavity I, although its volume was higher. For the sake of conciseness and due to the similitude of both cavities, no diagram is included for cavity II.

Four samples have been considered in the measurements, two of them were made of EPOMAR 2003-A epoxy resin $\left(\varepsilon^{*}=\right.$ $3.78-j 0.91)$ and the other two samples were made of CC polyester $\left(\varepsilon^{*}=4.66-j 0.4\right)$. Two shapes were considered for both materials, i.e., parallelepiped and cylindrical. In the case of parallelepiped samples, their longest sizes were always placed parallel to the $x$-axis. For a complete description of the samples' geometry, see Table I.

In order to measure the efficiency of the cavity, the $S_{11}$ complex scattering parameter was acquired at evenly spaced load 


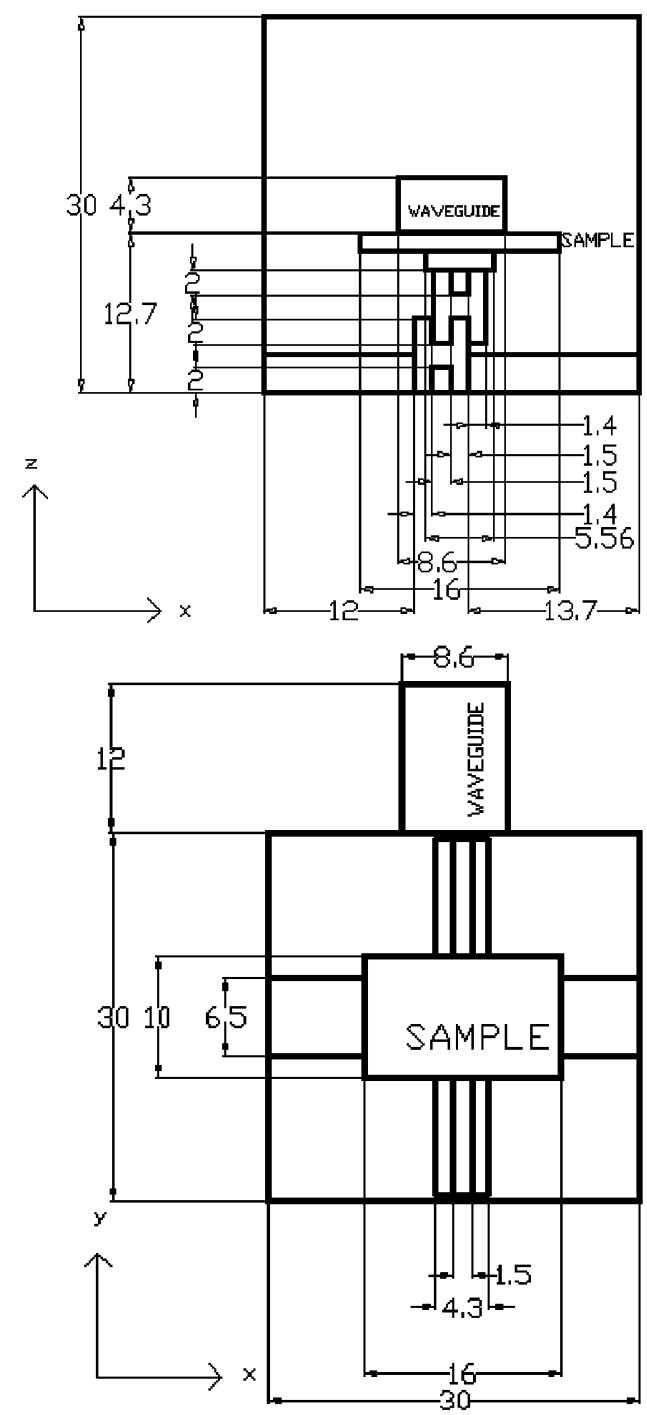

Fig. 1. Scheme of the multimode cavity and Styrofoam supporting. Dimensions in centimeters.

TABLE I

SAMPLE FEATURES

\begin{tabular}{lll}
\hline \hline Sample & MATERIAL -SHAPE & Dimensions $(\mathrm{cm})$ \\
\hline$A$ & Polyester - Cylindrical & Radius $=2.85 \mathrm{~cm}, \mathrm{z}=2 \mathrm{~cm}$ \\
$B$ & Epoxy - Cylindrical & Radius $=2.96 \mathrm{~cm}, \mathrm{z}=2 \mathrm{~cm}$ \\
$C$ & Polyester- Parallelepiped & $\mathrm{x}=16 \mathrm{~cm}, \mathrm{y}=10 \mathrm{~cm}, \mathrm{z}=1.4 \mathrm{~cm}$ \\
$D$ & Epoxy - Parallelepiped & $\mathrm{x}=19 \mathrm{~cm}, \mathrm{y}=10 \mathrm{~cm}, \mathrm{z}=0.86 \mathrm{~cm}$ \\
\hline
\end{tabular}

locations along the $y$-axis. Power efficiency can be readily inferred from $S_{11}$, as described in [10]

$$
\eta=1-\left|S_{11}\right|^{2}
$$

An RS-Amidata V-8756 reversible steeper motor of $2 \mathrm{~W}$ power consumption with 48 steps per revolution was controlled with a personal computer (PC) to exactly locate the sample along the $y$-axis. A ZVM Rohde\&Swarz vector network analyzer (VNA) was used to measure $S_{11}$ at each sample location for the frequency range $(2.4$ and $2.6 \mathrm{GHz})$.

\section{B. LM Optimization Method}

In this study, the LM method [11] has been employed to find the optimum position for the dielectric load that ensures a minimum energy reflection in the waveguide feeding. In order to carry out the optimization process, $N=10$ initial values taken from the $S_{11}$ response provided by the VNA have been obtained by locating the sample in equidistant points along the $y$-axis.

It must be remarked that, although the LM algorithm ensures an optimal solution for the model, it could possibly not be the best solution, but a local minimum. Thus, one of the critical aspects of this algorithm is the initial point where to begin the iterative optimization process. In this study, the $y$ location of the $N$ measured samples, which has the minimal value for $\left|S_{11}\right|$, has been chosen as the initial coordinate for the LM algorithm.

The LM algorithm is designed for the minimization of the error vector in (2) by iteratively updating the weights of a function that approximates the considered process. Thus, if $\vec{\omega}_{\text {new }}$ and $\vec{\omega}_{\text {old }}$ represent the current and final values of the weights vector in each iteration, the error vector for all the samples can be expanded in a Taylor series in the following way:

$$
\operatorname{Er} \vec{r}\left(\vec{\omega}_{\text {new }}\right)=\operatorname{Er} \vec{r}\left(\vec{\omega}_{\text {old }}\right)+Z\left(\vec{\omega}_{\text {new }}-\vec{\omega}_{\text {old }}\right)
$$

where $Z$ is a matrix with elements

$$
Z_{i j}=\frac{\partial \operatorname{Err}(i)}{\partial \omega_{j}}
$$

and $\omega_{j}$ is the $j$ th weight vector component.

The expression used to update the weights of the considered function can be obtained by the minimization of the error vector with respect to the weights

$$
\omega_{\text {new }}=\omega_{\text {old }}-\left(Z^{T} Z\right)^{-1} Z^{T} \operatorname{Err}\left(\vec{\omega}_{\text {old }}\right)
$$

where the term $Z^{T} Z$ can be considered as an approach to the Hessian matrix $H$.

In order to control the convergence step of the LM algorithm, the so-called $\lambda$ parameter is included in (4), resulting in the general expression

$$
\omega_{\text {new }}=\omega_{\text {old }}-(H+\lambda I)^{-1} Z^{T} \operatorname{Er} \vec{r}\left(\vec{\omega}_{\text {old }}\right) .
$$

By applying the LM algorithm to the proposed microwave applicators, the error function at the $n$th iteration that has to be minimized can be expressed as a one-dimensional function given by

$$
\operatorname{Err}(n)=\left|S_{11}(n)-0\right|
$$

where the zero value is the desired optimal value for $\left|S_{11}\right|$.

In this case, the $Z$ and $H$ matrix only contain one component with the gradient and Hessian values, respectively, which results in (7)-(10)

$$
\begin{aligned}
y(n+1) & =y(n)-(H(n)+\lambda)^{-1} G(n) \cdot \operatorname{Err}(n) \\
H(n) & =\frac{\partial^{2} \operatorname{Err}(n)}{\partial y^{2}} \\
G(n) & =\frac{\partial \operatorname{Err}(n)}{\partial y} \\
\operatorname{Err}(n) & =\left|S_{11}(n)\right|
\end{aligned}
$$




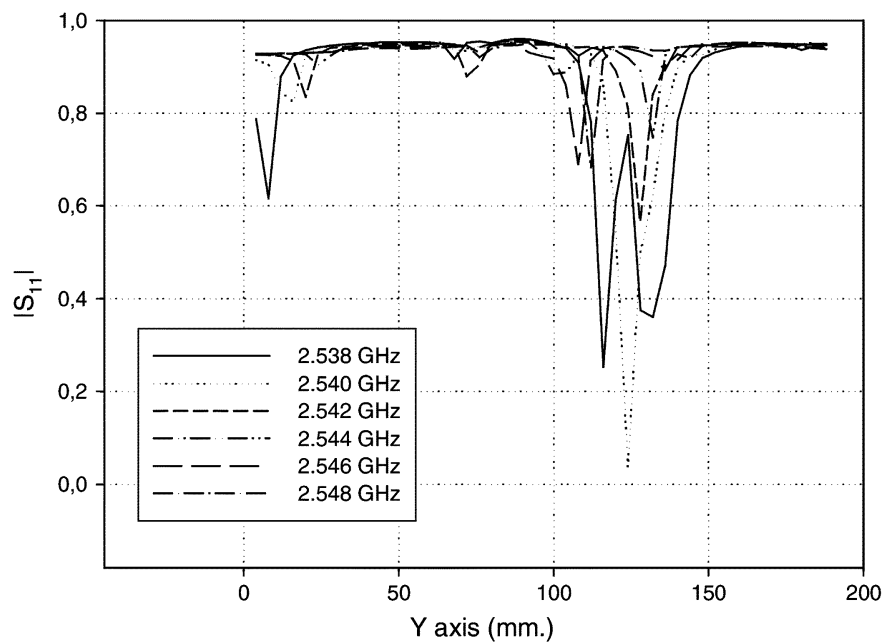

Fig. 2. Evolution of $\left|S_{11}\right|$ for the polyester cylinder (sample $A$ ) at different frequencies.

where $n$ represents the iteration number, $H$ is the Hessian component, $G$ is the gradient vector along the $y$-direction, $\lambda$ is a convergence parameter, which is updated at every iteration, and $\operatorname{Err}(n)$ is the error function calculated in the $n$th iteration. This error is compared with the desired solution (in this case, zero). Each iteration of the LM algorithm reduces the error until the predetermined error level is reached or a local minimum is found.

The LM optimization algorithm described above has been programmed and implemented in a PC, which stores the $\left|S_{11}\right|$ value for each sample position acquired from the VNA. This program calculates the next position of the sample that minimizes (10) and, finally, interacts through serial connection with the motor controller in order to place the sample in the position estimated by the LM algorithm. Therefore, the optimization algorithm is computed in the PC, but the rest of the measurements are experimentally obtained through the network analyzer.

Once the $y(n)$ optimum position was calculated through (7)-(10), the V-8756 motor was controlled in order to exactly position the sample at that location. The VNA then updated the measured $S_{11}$ at that position and (7)-(10) were iteratively computed to find the new optimum position $y(n+1)$.

\section{EXPERIMENTAL RESULTS}

\section{A. $S_{11}$ Measurements With Cavity I}

Four different samples $(A-D)$ have been introduced in cavity I in order to find their optimal position in terms of power efficiency. The influence of the operating frequency, the dimensions, and type of material have been analyzed for this oven.

Figs. 2 and 3 illustrate the $\left|S_{11}\right|$ values measured for different frequencies within a $10-\mathrm{MHz}$ bandwidth at discrete load locations with 4-mm intervals along the $y$-axis for samples $A$ and $B$, respectively.

From Fig. 2, one can observe the existence of an optimal position for the $A$ sample around $y=125 \mathrm{~mm}$. It can be appreciated that this optimum position is very dependant on the operating frequency. On the other hand, Fig. 2 shows

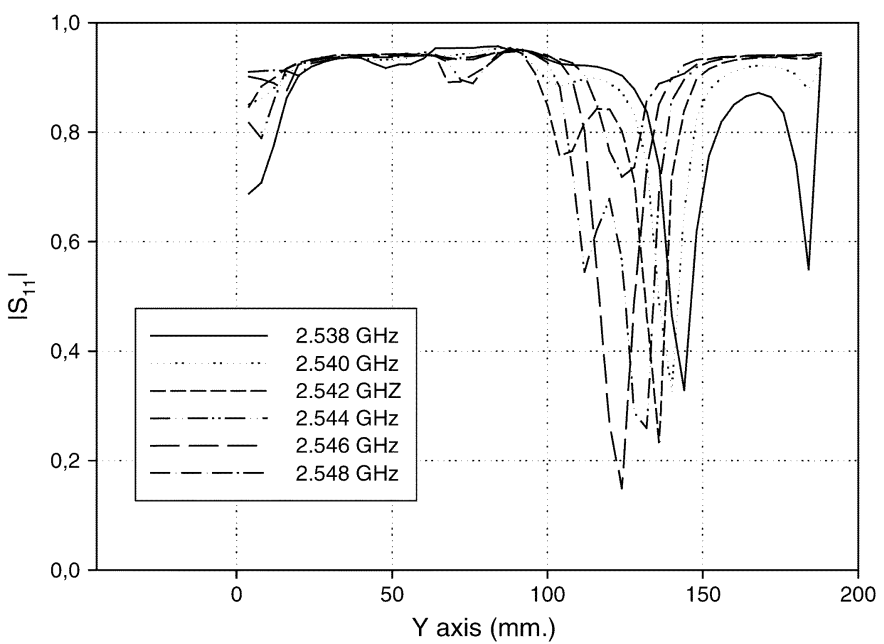

Fig. 3. Evolution of $\left|S_{11}\right|$ for the epoxy cylinder (sample $B$ ) at different frequencies.

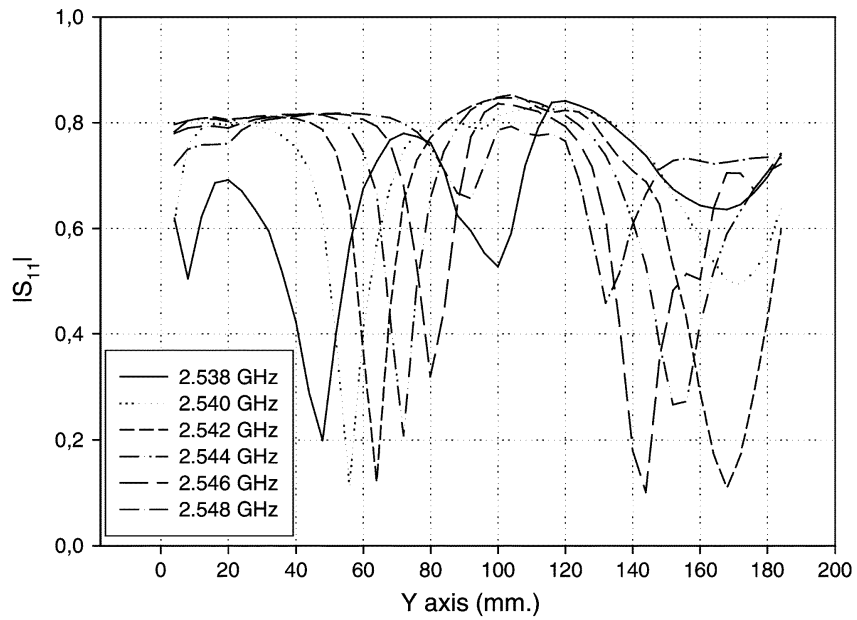

Fig. 4. Evolution of $\left|S_{11}\right|$ for the polyester parallelepiped (sample $C$ ) at different frequencies.

that important matching values can be achieved $\left(\left|S_{11}\right|<0.1\right)$ provided that the sample is properly placed inside the cavity. In fact, the inappropriate positioning of the sample may lead to values of $\left|S_{11}\right|$ higher than 0.9 . Similar results can be observed for the $B$ sample from Fig. 3 .

The same type of measurements have been carried out for samples $C$ and $D$. Figs. 4 and 5 show the $\left|S_{11}\right|$ evolution for several locations at the $y$-axis and several frequencies. In this case, both sample $C$ and $D$ show optimum positions around $y=60 \mathrm{~mm}$ and $y=150 \mathrm{~mm}$. $\left|S_{11}\right|$ reaches values around 0.2 for these sample locations, therefore increasing the power efficiency. Again, this optimum value shows a great dependence on the analyzed frequency.

The comparison of Figs. 2-5 shows that the behavior of $\left|S_{11}\right|$ is very similar for samples $A-D$ despite their different sizes, and confirms that the shape of the sample is of utmost importance to find the most efficient position within the multimode cavity. In fact, from these figures, one can conclude that samples $A$ and $B$ show a minimum value for $\left|S_{11}\right|$ around $120 \mathrm{~mm}$ at the $y$-axis, while the $C$ and $D$ samples show two areas in which minimum 


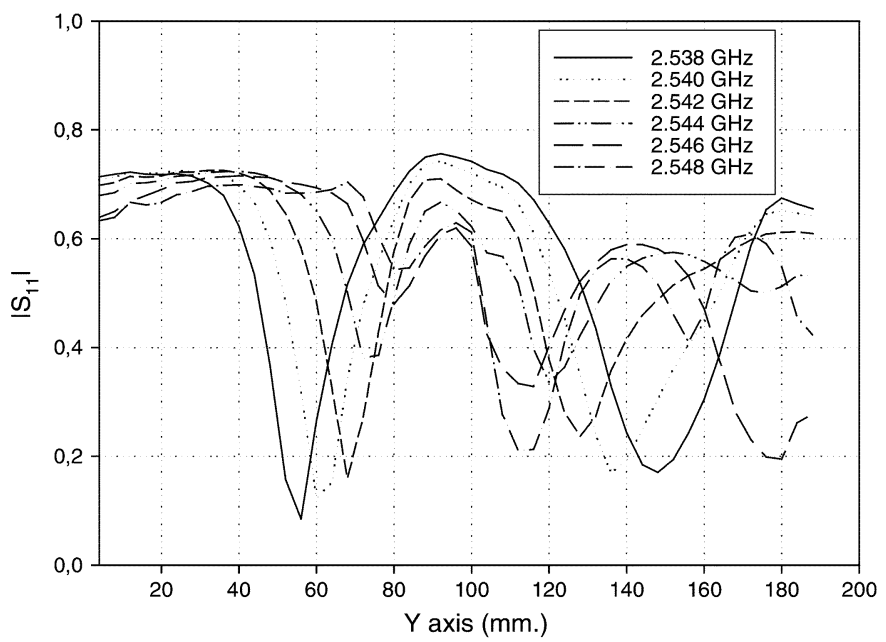

Fig. 5. Evolution of $\left|S_{11}\right|$ for the epoxy parallelepiped (sample $D$ ) at different frequencies.

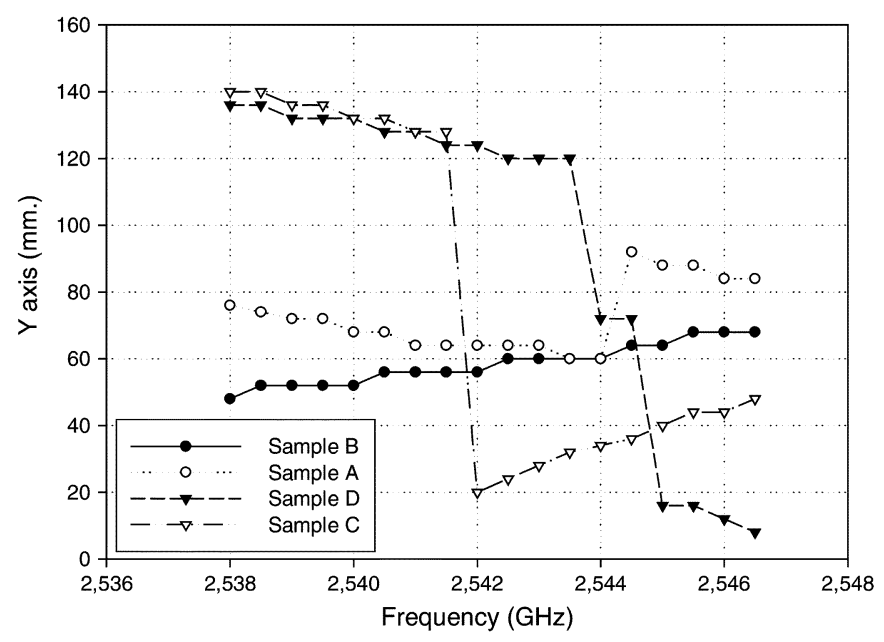

Fig. 6. Evolution of the optimal position ( $y$-axis) versus frequency for samples $A-D$.

values are localized (around 60 and $150 \mathrm{~mm}$ at the $y$-axis). Additionally, those optimum locations seem to vary linearly with frequency.

Due to the similar behavior found in the evolution of the optimum location for both the cylindrical $(A, B)$ and parallelepiped $(C, D)$ dielectric samples, the optimum location has been analyzed as a function of the frequency for all the considered samples. Fig. 6 shows the optimum location of each of the samples versus the analyzed frequency in the range ( 2.538 and $2.547 \mathrm{GHz}$ ). It can be observed that, for certain frequency ranges, the optimum sample location agrees for both cylindrical and parallelepiped samples, which is more evident for parallelepiped ones.

Additionally, a lineal displacement can be found for the optimal sample position as a function of frequency. However, these lineal displacements are interrupted with abrupt changes in the optimal position, mainly for samples $\mathrm{C}$ and $\mathrm{D}$. Although not completely, these results are in concordance with the conclusions reported in [10].

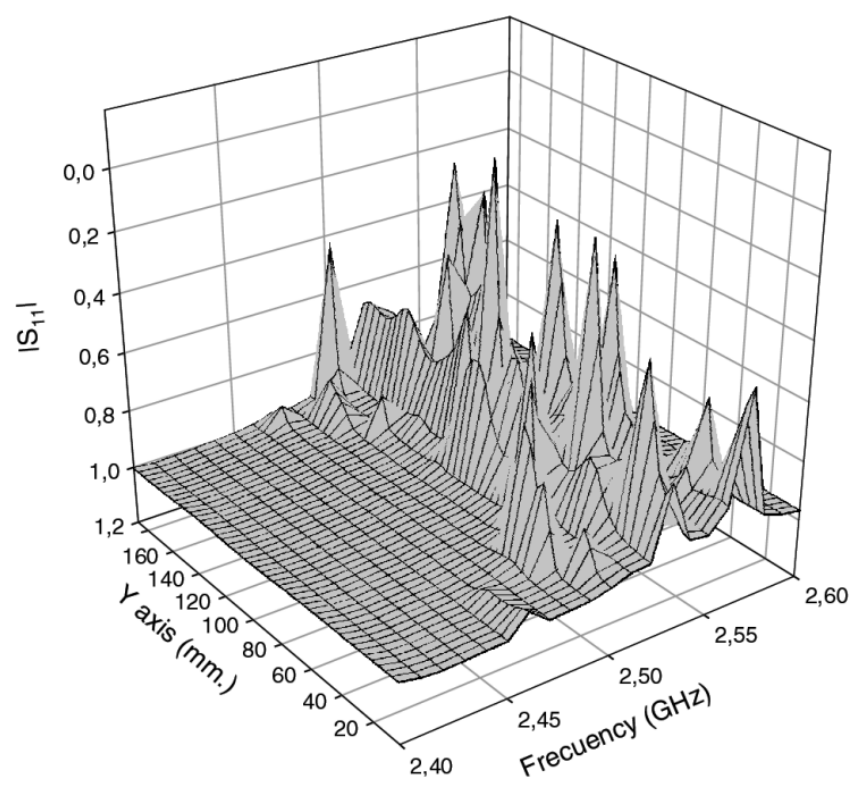

Fig. 7. $\left|S_{11}\right|$ versus sample position measured for a $200-\mathrm{MHz}$ bandwidth and sample $A$.

In order to broaden the study of this optimization procedure to a wider bandwidth, the reflection parameter $\left|S_{11}\right|$ has been measured between 2.4-2.6 GHz. Fig. 7 shows a two-dimensional plot of $\left|S_{11}\right|$ for sample $A$ versus the sample location at the $y$-axis and frequency. From this figure, one can conclude that $\left|S_{11}\right|$ oscillates more at a higher frequency and that no optimum position can be found below $2.50 \mathrm{GHz}$.

The explanation for this behavior can be found in [12]. From this study, one can conclude that if we consider the $30 \times 30 \times 30 \mathrm{~cm}^{3}$ cavity as a short-circuited waveguide (with a $30 \times 30 \mathrm{~cm}^{2}$ section), $2.50 \mathrm{GHz}$ is the degenerate cutoff frequency for the four TE and TM modes with indices 3 and 4. In this study, it is also concluded that match at or near TM mode cutoff frequencies cannot be achieved with ordinary tuning elements.

Since load matching performance is good just above the TM cutoff frequency for indices 3 and 4, the simplest solution for achieving good efficiency values within the industrial-scientific-medical (ISM) band would be to change cavity I dimensions to force these TM cutoff frequencies to be just below $2.4 \mathrm{GHz}$. In this case, increasing dimensions approximately by the factor of $2.55 / 2.45$ would provide a cubical cavity of $31.22 \times 31.22 \times 31.22 \mathrm{~cm}^{3}$ with TM cutoff frequencies around $2.4 \mathrm{GHz}$ for indices 3 and 4 . This cavity scaling would be enough to extrapolate the obtained matching results in the 2.5-2.6-GHz band to the ISM band.

\section{B. $S_{11}$ Measurements With Cavity II}

In order to demonstrate that the proposed load matching method can be applied for the ISM band placed at $2.4-2.5 \mathrm{GHz}$, different reflection measurements have been carried out on a $60 \times 60 \times 60 \mathrm{~cm}^{3}$ multimode oven (cavity II).

Fig. 8 illustrates $\left|S_{11}\right|$ measured for different frequencies within a $100-\mathrm{MHz}$ bandwidth around the microwave heating central frequency $(2.45 \mathrm{GHz})$. Sample $B$ was placed at discrete load locations with 8 -mm intervals along the $y$-axis of cavity II. 


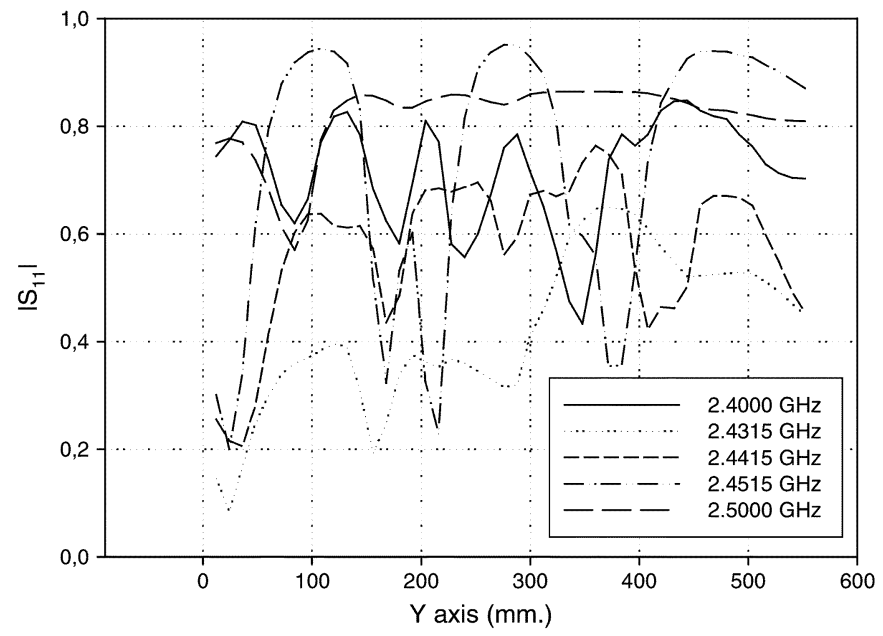

Fig. 8. Evolution of $\left|S_{11}\right|$ for sample $B$ at different frequencies and sample locations.

In Fig. 8, one can observe the existence of several optimal positions for the $B$ sample, being that these locations are very dependant on the operating frequency. Again, good matching values can be achieved $\left(\left|S_{11}\right|<0.3\right)$ provided that the sample is properly placed inside the cavity.

Several conclusions can be obtained by comparing the behavior of $\left|S_{11}\right|$ both in cavity I and cavity II. In fact, cavity II shows more oscillations for this magnitude as a function of the sample location than cavity I. This may be explained by the higher number of modes present in cavity II versus the ones that resonate in cavity I.

Additionally, a clear relationship between optimum location displacement and the operating frequency has not been found for measurements in cavity II.

\section{LM Optimization Results}

The described optimization algorithm has been implemented in the experimental platform consisting of the VNA, a PC, and the V-8756 motor. The aim of this study was to minimize the initial number of positions to be measured.

From previous trials, it was shown that $N=10$ initial samples were enough to obtain good optimization results. The initial value for $\lambda$ in (7) was set to 0.1 , while the factor for online modifying this parameters was 10, as described in [11].

The first iteration of the proposed algorithm always started at the sample location whose $\left|S_{11}\right|$ measured value was lowest. From this initial value, (7)-(10) were applied to update the sample location and to control the V-8756 motor.

The results obtained for sample $A$ at $2.53 \mathrm{GHz}$ are shown in Fig. 9. There, the black dots represent the ten initially measured data. Grey dots show the evolution of the sample location during the optimization loop and the dotted line is a conventional radial basis function (RBF) interpolation [13] of the $\left|S_{11}\right|$ behavior versus the $y$ location. This interpolation function is only used for helping the graphical interpretation of the LM algorithm behavior. The nonlinear RBFs are based on the generation of signals by using Gaussian functions whose superposition allows the interpolation of points of a real curve from a few

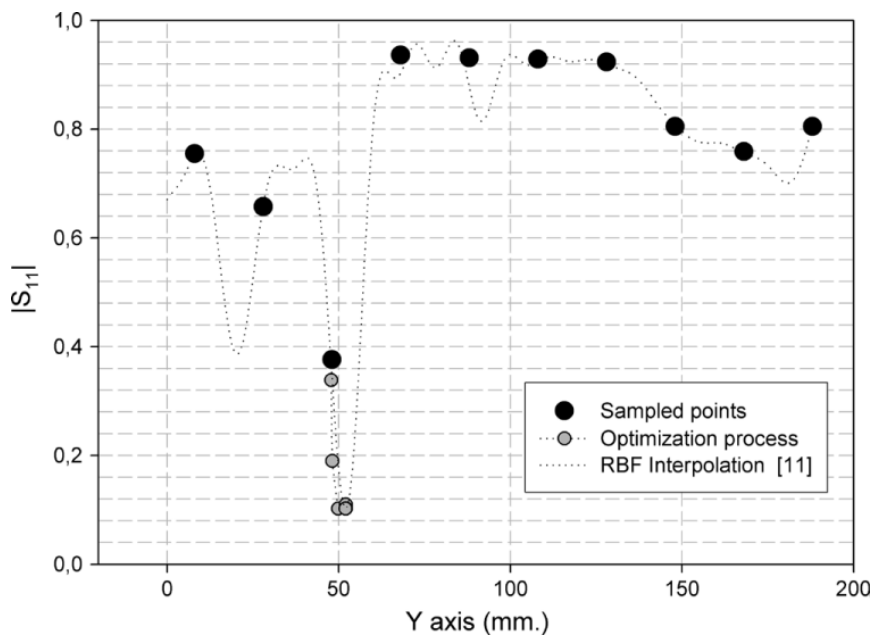

Fig. 9. Optimization procedure for sample $A$ at the frequency of $2.53 \mathrm{GHz}$ within cavity I.

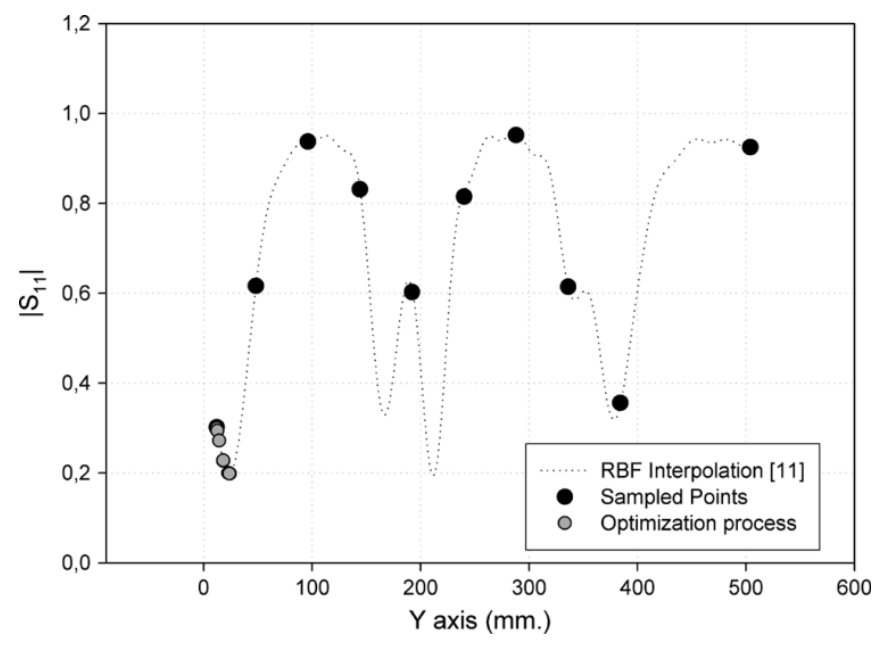

Fig. 10. Optimization procedure for sample $B$ at the frequency of $2.4415 \mathrm{GHz}$ within cavity II.

initial measured samples, obtaining excellent approximations of the real process.

From Fig. 9, one can conclude that four iterations are enough to find the best solution in terms of efficiency. In this case, $\left|S_{11}\right|$ reached an optimum value of 0.1024 , achieving a power efficiency around $99 \%$ at $y=52 \mathrm{~mm}$.

In the same way, this procedure has also been applied to cavity II and sample $B$ for an operating frequency equal to $2.4415 \mathrm{GHz}$. Fig. 10 shows the evolution of the sample $B$ location during the optimization loop. In this case, eight iterations were necessary for the LM algorithm to reach the optimum sample location within cavity II, $y=23.61 \mathrm{~mm}$, yielding an efficiency value of $96.05 \%\left(\left|S_{11}\right|=0.1987\right)$.

The convergence of the optimization procedure for both cavity I and II is more clearly appreciated in Fig. 11. It can be deduced from these data that the convergence is achieved in four iterations for cavity I, while it takes eight iterations for cavity II. If we add these values to the ten initial measurements, one can conclude that only 14 and 18 measurements are required to achieve a power efficiency of 99 and $96 \%$ for cavities I and II, respectively. 


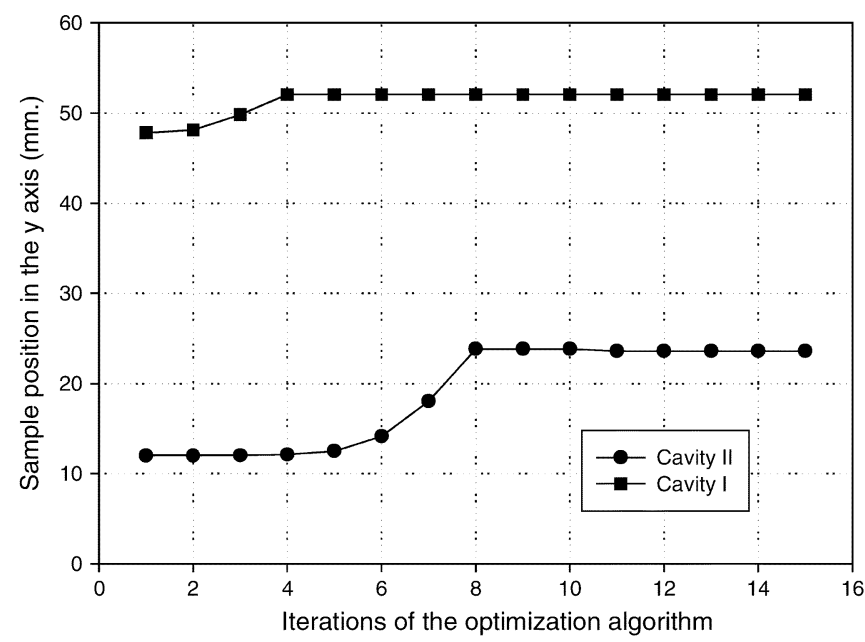

Fig. 11. Convergence of the optimization procedure for sample $A$ at $2.53 \mathrm{GHz}$.

Therefore, it can be concluded from these data that the time needed to carry out the optimization process is very dependant on the operating conditions: 1) cavity and sample dimensions; 2) sample dielectric properties; and 3) operating frequency since these parameters influence the behavior of $\left|S_{11}\right|$ versus the sample location.

\section{CONCLUSION}

A novel multimode cavity load-matching method has been experimentally validated for several samples with different permittivities and shapes and two different multimode cavities. The results show that the relationship between the operating frequency and cavity dimensions is critical. In fact, measurements demonstrate that high power efficiencies can be obtained provided that operating frequency is not just below TM mode cutoff frequencies for the transverse dimensions of the oven. Additionally, the load-matching method has shown a great sensitivity to the used frequency, which demands an accurate knowledge of the operating frequency for the microwave sources. In fact, a quasi-linear relationship for the optimum location displacement and the operating frequency has been found for cavity I, while this has not been possible for cavity II.

The LM method has been successfully employed to locate a dielectric sample within the multimode cavity achieving a power efficiency around $99 \%$.

It must be remarked that this novel load matching method does not take into account the electric-field patterns that would appear within the dielectric samples and, consequently, their temperature uniformity, which are important magnitudes that influence microwave heating quality. In fact, such a study was out of the scope of this paper, although further research is envisaged in this direction.

Finally, one can easily conclude from measured data that dielectric changes within the samples would lead to optimum position changes. As a result, in industrial microwave heating systems, in which high temperature variations are expected within the dielectric materials, both the samples' permittivity and their optimum position would change during the process. In this case, a continuous real-time monitoring process for power efficiency would be necessary to adaptively compute the optimum sample position and to relocate the sample.

\section{REFERENCES}

[1] J. M. Osepchuk, "Microwave power applications," IEEE Trans. Microw. Theory Tech., vol. 50, pp. 975-985, Mar. 2002.

[2] A. C. Metaxas, "Radiofrequency and microwave heating: A perspective for the millennium," Power Eng. J., vol. 14, pp. 51-60, 2000.

[3] A. C. Metaxas and R. J. Meredith, Industrial Microwave Heating. London, U.K.: Peregrinus, 1983.

[4] R. G. Meredith, Engineers' Handbook of Industrial Microwave Heating. London, U.K.: IEE, 1998.

[5] T. V. Chow-Ting-Chan and H. C. Reader, Understanding Microwave Heating Cavities. London, U.K.: Artech House, 2000, pp. 126-163.

[6] V. V. Yakovlev, "Comparative analysis of contemporary EM software for microwave power industry," Microwaves: Theory and Applications in Material Processing V. Ceramic Transactions, vol. 111, pp. 551-558, 2000.

[7] E. Murphy and V. V. Yakovlev, "FDTD-backed RBF network technique for efficiency optimization of microwave structures," in Proc. 9th AMPERE Microwave and High Frequency Heating Conf., Loughborough, U.K., Sep. 2003, pp. 197-200.

[8] J. Monzó-Cabrera, A. Díaz-Morcillo, J. L. Pedreño-Molina, and D. Sánchez-Hernández, "A new method for load matching in multimode-microwave heating applicators based on the use of dielectric-layer superposition," Microw. Opt. Technol. Lett., vol. 40, no. 4, pp. 318-322, 2004.

[9] J. Monzó-Cabrera, J. Escalante, A. Díaz-Morcillo, A. MartínezGonzález, and D. Sánchez-Hernández, "Load matching in multimode microwave-heating applicators based on the use of dielectric-layer with commercial materials," Microw. Opt. Technol. Lett., vol. 41, no. 5, pp. 414-417, 2004.

[10] M. E. Requena-Pérez, J. L. Pedreño-Molina, M. Pinzolas-Prado, J. Monzó-Cabrera, A. Díaz-Morcillo, and D. Sánchez-Hernández, "Load matching in multimode microwave-heating applicators by load location optimization," in Proc. 34th Eur. Microwave Conf., Amsterdam, The Netherlands, 2004, pp. 1549-1552.

[11] C. M. Bishop, Neural Network for Pattern Recognition. Oxford, U.K. Oxford Univ. Press, 2004.

[12] J. M. Osepchuk, "Microwave heating," Wiley Encyclopedia of Electrical and Electronics Engineering, vol. 13, pp. 118-127, 1999.

[13] D. Broomhead and D. Lowe, "Multivariable functional interpolation and adaptive networks," Complex Syst., vol. 2, pp. 322-355, 1988.

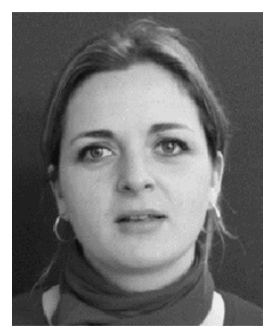

María E. Requena-Pérez was born in Alicante, Spain. She received the Dipl. Ing. degree in telecommunications engineering from the Universidad Politécnica de Valencia, Valencia, Spain, in 2001, and is currently working toward the Ph.D. degree at the Universidad Politécnica de Cartagena, Cartagena, Spain.

In 2003, she joined the Departmento de Tecnologías de la Información y las Comunicaciones, Universidad Politécnica de Cartagena. Her current research areas cover microwave-assisted heating and drying processes, microwave applicator design, permittivity measurement and optimization and numerical techniques in electromagnetics.

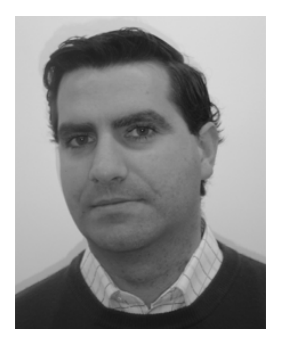

Juan L. Pedreño-Molina was born in Cartagena (Murcia), Spain, in 1969. He received the B.A.Sc. degree and Ph.D. degree in neurotechnology, control, and robotics from the Technical University of Madrid (UPM), Madrid, Spain, in 1984 and 2000, respectively.

He is currently an Assistant Professor of telecommunication engineering with the Universidad Politécnica de Cartagena (UPCT), Cartagena, Spain. Since 1999, he has been with the Departmento de Tecnologías de la Información y las Comunicaciones, UPCT. His research interests are in signal processing applied to optimization and control of nonlinear systems and modeling based on neural networks with applications to robotics and drying processes. 


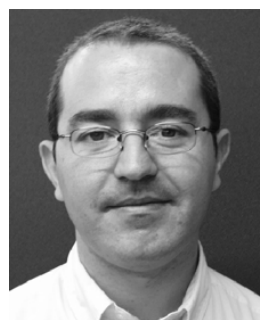

Juan Monzó-Cabrera was born in Elda (Alicante), Spain, on January 1973. He received the Dipl. Ing. and Ph.D. degrees in telecommunications engineering from the Universidad Politécnica de Valencia, Valencia, Spain, in 1998 and 2002, respectively.

From 1999 to 2000, he was a Research Assistant with the Microwave Heating Group (GCM). In 2000, he joined the Departamento de Teoría de la Señal y Radiocomunicaciones, Universidad Politécnica de Cartagena, Cartagena, Spain, as an Associate Lecturer. He is currently an Associate Lecturer with the Departamento de Tecnologías de la Información y Comunicaciones, Universidad Politécnica de Cartagena. He has coauthored over 30 papers in refereed journals and conference proceedings. He holds one patent regarding microwave heating industrial processes. His current research areas cover microwave-assisted heating and drying processes, microwave applicator design and optimization, and numerical techniques in electromagnetics. He is a reviewer of several international journals.

Dr. Monzó-Cabrera is a member of the Association of Microwave Power in Europe for Research and Education (AMPERE), a European-based organization devoted to the promotion of RF and microwave energy.

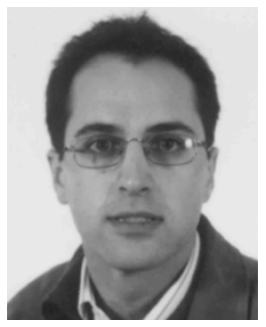

Alejandro Díaz-Morcillo (S'95-M'02) was born in Albacete, Spain, in 1971. He received the Ingeniero (M.S.Eng.) and Doctor Ingeniero (Ph.D.) degrees in telecommunication engineering from the Universidad Politécnica de Valencia (UPV), Valencia Spain, in 1995 and 2000, respectively.

From 1996 to 1999, he was a Research Assistant with the Departmento de Comunicaciones, UPV. In 1999, he joined the Departmento de Tecnologías de la Información y las Comunicaciones, Universidad Politécnica de Cartagena (UPCT), Cartagena, Spain, as a Teaching Assistant. In 2001, he became an Associate Professor with UPCT. He leads the Electromagnetics and Matter Research Group, UPCT. His main research interest are numerical methods in electromagnetics and industrial microwave heating systems. 